\title{
Cigarette smoking in patients with obsessive compulsive disorder: a report from the International College of Obsessive Compulsive Spectrum Disorders (ICOCS)
}

\author{
Bernardo Dell'Osso, ${ }^{1,2 *}$ Humberto Nicolini, ${ }^{3,4}$ Nuria Lanzagorta, ${ }^{4}$ Beatrice Benatti, ${ }^{1}$ \\ Gregorio Spagnolin, ${ }^{1}$ M. Carlotta Palazzo, ${ }^{\prime}$ Donatella Marazziti, ${ }^{5}$ Eric Hollander, ${ }^{6}$ \\ Naomi Fineberg, ${ }^{7}$ Dan J. Stein, ${ }^{8}$ Stefano Pallanti, ${ }^{9}$ Michael Van Ameringen, ${ }^{10}$ Christine \\ Lochner, ${ }^{11}$ Georgi Hranov, ${ }^{12}$ Oguz Karamustafalioglu, ${ }^{13}$ Luchezar Hranov, ${ }^{12}$ Joseph \\ Zohar,${ }^{14}$ Damiaan Denys, ${ }^{15}$ A. Carlo Altamura, ${ }^{1}$ and Jose M. Menchon ${ }^{16}$
}

\footnotetext{
${ }^{1}$ Department of Psychiatry, University of Milan, Fondazione IRCCS Ca'Granda, Ospedale Maggiore Policlinico, Milano, Italy

${ }^{2}$ Bipolar Disorders Clinic, Stanford University, Stanford, California, USA

${ }^{3}$ Carracci Medical Group, Mexico City, Mexico

${ }^{4}$ Center for Genomic Sciences, Universidad Autónoma de la Ciudad de México, Mexico City, Mexico

${ }^{5}$ Dipartimento di Psichiatria, Neurobiologia, Farmacologia e Biotechnologie, Università di Pisa, Pisa, Italy

${ }^{6}$ Department of Psychiatry and Behavioral Sciences, Albert Einstein College of Medicine and Montefiore Medical Center, New York, USA

${ }^{7}$ Mental Health Unit, Hertfordshire Partnership Foundation Trust, Queen Elizabeth II Hospital, Welwyn Garden City, UK

${ }^{8}$ MRC Unit on Anxiety and Stress Disorders, Department of Psychiatry and Mental Health, University of Cape Town, Cape Town, South Africa

${ }^{9}$ Department of Psychiatry, University of Florence, and Institute of Neurosciences, Florence, Italy

${ }^{10}$ Department of Psychiatry and Behavioural Neurosciences, McMaster University, Hamilton, Canada

${ }^{11}$ MRC Unit on Anxiety and Stress Disorders, Department of Psychiatry, University of Stellenbosch, South Africa

${ }^{12}$ University Multiprofile Hospital for Active Treatment in Neurology and Psychiatry Sveti Naum, Sofia, Bulgaria

${ }^{13}$ Department of Psychiatry, Sisli Eftal Teaching and Research Hospital, Istanbul, Turkey

${ }^{14}$ Department of Psychiatry, Chaim Sheba Medical Center, Tel Hashomer, Israel

${ }^{15}$ Department of Psychiatry, Academic Medical Center, University of Amsterdam, Amsterdam, the Netherlands

${ }^{16}$ Psychiatry Unit at Hospital Universitari de Bellvitge. Barcelona, Spain
}

Obsessive compulsive disorder (OCD) showed a lower prevalence of cigarette smoking compared to other psychiatric disorders in previous and recent reports. We assessed the prevalence and clinical correlates of the phenomenon in an international sample of 504 OCD patients recruited through the International College of Obsessive Compulsive Spectrum Disorders (ICOCS) network.

Cigarette smoking showed a cross-sectional prevalence of $24.4 \%$ in the sample, with significant differences across countries. Females were more represented among smoking patients $(16 \%$ vs $7 \%$; $p<.001)$. Patients with comorbid Tourette's syndrome $(\mathrm{p}<.05)$ and tic disorder $(\mathrm{p}<.05)$ were also more represented among smoking subjects. Former smokers reported a higher number of suicide attempts $(\mathrm{p}<.05)$.

We found a lower cross-sectional prevalence of smoking among OCD patients compared to findings from previous studies in patients with other psychiatric disorders but higher compared to previous and more recent OCD studies. Geographic differences were found and smoking was more common in females and comorbid Tourette's syndrome/tic disorder.

Received 26 June 2015; Accepted 20 July 2015

Key words: Cigarette smoking, comorbidity, gender, obsessive compulsive disorder (OCD), prevalence.

* Address for correspondence: Dr. Bernardo Dell'Osso, MD, Assistant Professor of Psychiatry, Department of Psychiatry, University of Milan, Fondazione IRCCS Ca' Granda, Ospedale Maggiore Policlinico, Via Francesco Sforza 35, 20122 Milano, Italy.

(Email: bernardo.dellosso@policlinico.mi.it)

The research was supported by the European College of Neuropsychopharmacology Research Networks Initiative. Funding for this study was provided within the framework of the collaboration of the International College of Obsessive Compulsive Spectrum Disorders (ICOCS). DJS and CL are supported by the MRC of South Africa.

We would like to thank all the collaborators of ICOCS worldwide (Bulgary, Canada, Italy, Israel, England, Mexico, Spain, South Africa, Turkey, and the United States of America).

Authors Bernardo Dell'Osso and Humberto Nicolini contributed equally to this article. 
TABLE 1. Socio-demographic and clinical characteristics of the study sample

\begin{tabular}{|c|c|c|c|c|}
\hline & $\operatorname{CSPs}(n=118,24.4 \%)$ & FSPs ( $n=96,19.9 \%)$ & $\operatorname{NSPS}(n=269,55.7 \%)$ & Total sample $(n=483)$ \\
\hline Mean age $\pm S D$ & $43.5 \pm 11.4$ & $43.5 \pm 11.6$ & $43.1 \pm 13.9$ & $43.3 \pm 13.1$ \\
\hline Age of onset $\pm S D$ & $20.2 \pm 10.9$ & $19.1 \pm 9.1$ & $19.2 \pm 10.9$ & $19.4 \pm 10.5$ \\
\hline Male:female & $7.5 \%: 16.9 \%^{* \star}$ & $12.4 \%: 7.4 \%{ }^{* *}$ & $20.1 \%: 35.6 \%{ }^{* *}$ & $39.9 \%: 60 \%$ \\
\hline History of suicide attempt & $13(2.7 \%)$ & $28(5.7 \%)^{*}$ & $23(4.7 \%)$ & $64(13.2 \%)$ \\
\hline Presence of medical comorbidity & $13(2.6 \%)$ & $30(6.2 \%)$ & $44(9.1 \%)^{* *}$ & $87(18 \%)$ \\
\hline Presence of psychiatric comorbidity & $14(2.8 \%)$ & $25(5.1 \%)$ & $49(10.1 \%)^{*}$ & $88(18.2 \%)$ \\
\hline Currently taking psychotropic medications & $66(13.6 \%)$ & $60(12.4 \%)$ & $191(39.5 \%)^{\star \star}$ & $317(65.6 \%)$ \\
\hline Previous psychiatric hospitalization & $17(3.5 \%)$ & $31(6.4 \%)$ & $34(7 \%)$ & $82(16.9 \%)$ \\
\hline Currently undergoing cognitive behavioral therapy & $56(11.6 \%)$ & $28(5.7 \%)$ & $102(21.2 \%)^{* *}$ & $186(38.5 \%)$ \\
\hline Y-BOCS score & $23.1 \pm 7.4$ & $22.7 \pm 7.5$ & $23.5 \pm 6.9$ & $23.1 \pm 7.8$ \\
\hline CGI-s score & $4.3 \pm 1.1$ & $4.1 \pm 2.6$ & $3.8 \pm 1.5$ & $4.0 \pm 1.3$ \\
\hline
\end{tabular}

Values for categorical and continuous variables are expressed as $\mathrm{N}(\%)$ and mean $\pm \mathrm{SD}$, respectively. Reported variables had a percentage of missing data ranging from $0 \%$ to $11 \%$. CSPs = currently smoking patients; FSPs = former smoking patients; NSPS = patients who had never smoked; Y-BOCS = Yale-Brown Obsessive Compulsive Scale; CGI-s $=$ Clinical Global Impression scale, severity of illness. ${ }^{*} p<.05,{ }^{* *} p<.001$.

Tobacco smoking is the most common substance use disorder in adults with mental illness, with rates at least twice as common as for general population. ${ }^{1}$ Lifetime smoking rate ranges between $50 \%$ and $80 \%$ in people with lifetime history of mental illness, ${ }^{1}$ whereas, for instance, a cross-sectional prevalence below $20 \%$ was reported for the general U.S. population. ${ }^{2}$

Mental disorders represent a risk factor for tobacco smoking, ${ }^{1}$ which, in turn, seems to be a negative prognostic factor for suicidal behavior, regardless of diagnosis. ${ }^{3}$

A few studies have investigated prevalence and clinical correlates of cigarette smoking in obsessive compulsive disorder (OCD) patients, reporting lower prevalence rates $(7.7 \%-22.4 \%)$, compared to what has been observed in patients with other psychiatric disorders and, likely, in the general population. ${ }^{4,5}$ Reasons for such data in OCD may be linked to illness-related factors, as well as biological and socio-environmental aspects.

Recently an American study reported a smoking rate of $13.3 \%$ in a sample of 113 OCD patients, with no difference in terms of demographics between smoking and non-smoking subjects, ${ }^{6}$ but a higher prevalence of impulsive features in smoking subjects.

Previously, studies assessing smoking prevalence and correlates in OCD had been conducted with limited national samples. It is well established, however, that smoking prevalence varies according to different countries, depending on multiple factors. ${ }^{3}$ Therefore, the present multicenter, international study aimed to investigate smoking prevalence and clinical correlates in a large sample of OCD patients, who were recruited from the ICOCS network through a naturalistic database collecting socio-demographic and clinical variables. ${ }^{7}$

The sample included 504 consecutive OCD outpatients attending different psychiatric services worldwide, participating in the ICOCS network. Details about involved centers and assessment procedures have been specified elsewhere. ${ }^{7}$

Data on cigarette smoking were collected during a clinical interview. For the purpose of the study, patients were divided in 3 smoking categories: current smoking patients (CSPs), former smoking patients (FSPs), and patients who had never smoked (NSPs). CSPs were defined as those whose consumption was at least 1 cigarette daily for the last 6 months.

After obtaining patients' written informed consent and approval from the local Ethics Committee/Institutional Review Board, the main socio-demographics, smokingrelated variables, and clinical features-psychometric evaluations [Yale-Brown Obsessive Compulsive Scale (Y-BOCS), Sheehan disability scale (SDS), MontgomeryAsberg Depression Rating Scale (MADRS), Clinical Global Impression scale-Severity of illness (CGI-s), Compulsive Personality Assessment Scale (CPAS)], comorbidity status (psychiatric/medical), lifetime suicide attempts and hospitalizations, current treatment (psychotropic medications and/or cognitive behavioral therapy [CBT])-were collected and analyzed for the sample and related subgroups using chi-squared test for categorical variables and $t$-tests for independent samples for continuous variables.

Data on smoking behavior accounted for $95.8 \%$ of the total sample $(\mathrm{n}=483)$. Female patients represented $60 \%$ of the sample. Socio-demographic and clinical data of the sample are summarized in Table 1.

The $24.4 \%$ of the total sample was represented by CSPs, while the $55.7 \%$ by NSPs.

Within the group of CSPs, there were significantly more females than males $(17 \%$ vs $7.4 \%$; $p<.001)$. Females were also significantly more represented than males in the subgroup of NSPs $(35.6 \%$ vs. $20.1 \%$; $\mathrm{p}<.001)$, whereas a male preponderance was observed in the group of FSPs $(12.4 \%$ vs $7.4 \%$; $<<.001)$. In both 
groups of CSPs and NSPs, the female proportion was approximately twice as prevalent as the male one.

With respect to prevalence differences according to geographic areas, the Italian sample had the highest number of NSPs, while the South African sample the highest number of CSPs $(p<.001)$.

With respect to psychiatric comorbidities, $18.2 \%$ of the total sample was found to have a comorbid disorder, with a significantly higher rate among NSPs $(10.1 \%$ in NSPs vs $5.1 \%$ in FSPs vs $2.8 \%$ in CSPs; $p<.05$ ). Considering specific comorbidity patterns, among patients with comorbid Tourette's syndrome and tic disorder, CSPs were significantly more represented than FSPs and NSPs $(\mathrm{p}<.05$; Figure 1$)$.

NSPs were found to show a more frequent history of previous psychiatric hospitalization (7\% in NSPs vs $6.4 \%$ in FSPs vs $3.5 \%$ in CSPs) compared to the other groups, even though not at a statistically significant level.

With respect to previous suicide attempts, a positive history was significantly more common in the group of FSPs $(p<.05)$.

With respect to the severity of illness, mean Y-BOCS scores among the 3 subgroups did not show any statistically significant differences, similarly to CGI-s scores.

With respect to treatment status, the $65.6 \%$ of the total sample was under pharmacological treatment at the time of the assessment, with a significantly higher rate of patients on psychotropic medications among NSPs than among FSPs and CSPs (39\% in NSPs vs $12.4 \%$ in FSPs vs $13.6 \%$ in CSPs; $\mathrm{p}<.05)$.

The $38.5 \%$ of the total sample was receiving CBT, with this form of psychotherapy being used more among
NSPs $(21.2 \%$ in NSPs vs $5.7 \%$ in FSPs vs $11.6 \%$ in CSPs; $\mathrm{p}<.001)$.

It is well established that smoking behavior is profoundly influenced by several factors, including biological and sociocultural aspects. In this respect, the geographic heterogeneity of our large, international sample might have minimized the risk of possible sociocultural bias and other conditioning factors related to a single catchment area. In fact, the ICOCS network involved patients from 3 continents and 10 different countries, thus providing a putatively representative estimate of cigarette smoking in OCD patients.

Of the total sample, $24.4 \%$ was found to be currently smoking, with a significantly higher rate among female patients. Such a finding seems to be only slightly higher than that observed in the general American population, but much lower compared to what has been previously reported in patients with psychiatric disorders. ${ }^{1}$ In this respect, the hypothesis that the prevalence of cigarette smoking in people with psychiatric disorders may be related to the neurochemical effects of nicotine in the orbitofrontal cortex seems to be supported by the present findings, suggesting that OCD may be positioned on one end of a continuum while schizophrenia is on the opposite one. ${ }^{5}$

Comparing our prevalence finding with previous studies (reporting rates ranging between $7 \%$ and $22.4 \%),{ }^{4-6}$ we found an overall higher rate in our sample. However, we also found significant differences across countries, which may be, in turn, due to several factors, including sample size, gender composition, and cultural issues.

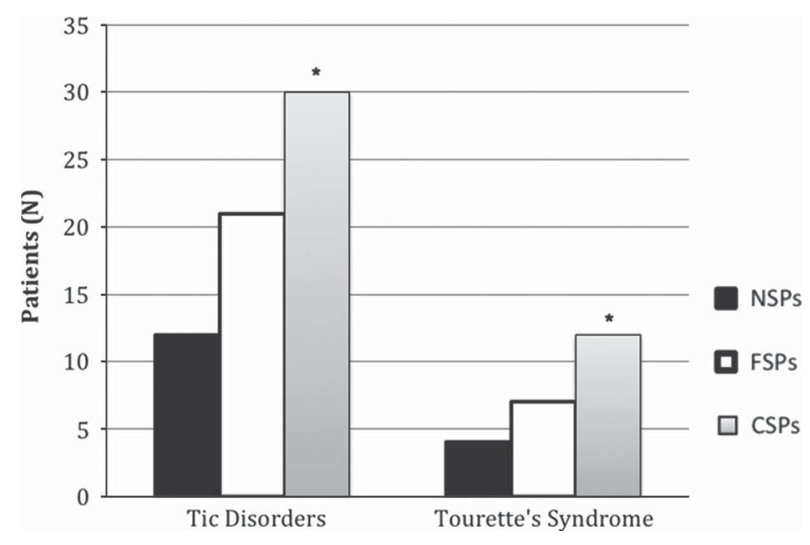

Legend:

NSPs: Patients who had never smoked

FSPs: Former Smoking Patients

CSPs: Currently Smoking Patients

Statistics:

Tourette's Syndrome: $x^{2}=20.06, p=0.01$; Tic Disorder: $x^{2}=17.48, p=0.02$.

FIGURE 1. Smoking habit among OCD patients with comorbid tic disorder and Tourette's syndrome. NSPs: patients who had never smoked; FSPs: former smoking patients; CSPs: currently smoking patients. Tourette's syndrome: $\chi^{2}=20.06, p=0.01$; tic disorder: $\chi^{2}=17.48, p=0.02$. 
With respect to gender aspects, our finding of a female preponderance in CSPs is not of univocal interpretation for different reasons. The first reason is because a similar gender rate was observed in NSPs. Second, the whole sample actually showed a female preponderance, different from what has been reported by the majority of previous studies. ${ }^{4}$ Third, the finding of male preponderance among FSPs, though consistent with most studies in the field that have shown that female gender represents a protective factor against smoking, ${ }^{2}$ further complicates the interpretation of gender data, warranting additional investigation.

With respect to psychiatric comorbidity, in our sample, the only comorbid conditions more frequently associated with smoking were Tourette's syndrome and tic disorder. Interestingly, general psychiatric comorbidity rates appeared to be lower in our sample (18.2\%) compared to other studies, ${ }^{4,6}$ potentially explaining the lack of other significant associations. Of note, a recent study investigating comorbidity between impulsive spectrum disorders and nicotine dependence suggested that impulsivity could represent the shared mechanism between pathological behavior and substance addictions including nicotine dependence. ${ }^{8}$ Another recent study found that smoking OCD patients showed higher impulsivity levels, reinforcing this assumption. ${ }^{6}$ In this perspective, our finding of higher prevalence of CSPs among patients with Tourette's syndrome and tic disorder may be explained in light of the fact that the aforementioned conditions are characterized by impulsive features, having been included within a common spectrum of disorders that share specific psychopathological bases and treatment-response aspects. ${ }^{9}$

With respect to previous suicide attempts, a prevalence of $13 \%$ in the whole sample was found, stressing that suicidal behaviors in OCD are not infrequent, though the literature in the field is controversial in such regard. ${ }^{10}$ In our sample, in particular, suicide attempts were significantly more numerous among FSPs. The finding of a higher vulnerability to suicidal behaviors in FSPs has been already reported ${ }^{3}$ and may be, in turn, associated with impulsive features. In addition, it is well established that smoking represents an independent risk factor for suicide in patients suffering from other psychiatric disorders. ${ }^{3}$ Thus, even though a common link between suicidality, impulsivity, and nicotine exposure may be hypothesized, further investigation exploring biological correlates of such a relation is warranted.

The findings showing no difference in terms of severity of illness among smoking and non-smoking patients seem to exclude an association between smoking and severity of illness in OCD patients, as also previously shown. ${ }^{6}$

Finally, we found that approximately two-thirds of the sample was receiving a pharmacological treatment, and that the presence of any treatment (either pharmacologic or psychotherapeutic) was significantly more represented among NSPs. Given that, the opposite finding (showing a greater frequency of lack of treatment in CSPs), as a potential explanation, was not statistically significant, likely due to sub-sample size limitation; additional investigation in the field is required.

The following methodological limitations should be considered when interpreting the reported results. Geographic prevalence data of cigarette smoking in the general population of involved centers were not considered, except for the U.S. Therefore, notwithstanding the international catchment, recruitment-related factors may have influenced reported results. In addition, the present study had a cross-sectional perspective, with longitudinal data being likely more informative.

\section{Disclosures}

Bernardo Dell'Osso has the following disclosures: AstraZeneca, Speaker's Bureau, Speaker's fee; Bristol Myers Squibb, Speaker's Bureau, Speaker's fee; Janssen-Cilag, Speaker's Bureau, Speaker's fee; Eli Lilly, Speaker's Bureau, Speaker's fee; Pfizer, Speaker's Bureau, Speaker's fee; GlaxoSmithKline, Speaker's Bureau, Speaker's fee Lundbeck, Speaker's Bureau, Speaker's fee; Cyberonics, Speaker's Bureau, Speaker's fee; Italfarmaco, Speaker's Bureau, Speaker's fee; Roche, Consultant, Honoraria; Merck, Consultant, Honoraria; AstraZeneca, Consultant, Honoraria; BristolMyers Squibb, Consultant, Honoraria; Janssen Cilag, Consultant, Honoraria; Lundbeck, Consultant, Honoraria; Sanofi, Consultant, Honoraria; Eli Lilly, Consultant, Honoraria; Pfizer, Consultant, Honoraria.

Humberto Nicolini has the following disclosures: Forum Pharmaceuticals, Principal Investigator, Research support.

Nuria Lanzagorta does not have anything to disclose.

Beatrice Benatti does not have anything to disclose.

Gregorio Spagnolin does not have anything to disclose.

M. Carlotta Palazzo does not have anything to disclose.

Donatella Marazziti does not have anything to disclose.

Eric Hollander has the following disclosures: Sunovion, Speakers Bureau, Speaker's fee; Shire, Consultant, Consulting fee; Roche, Researcher, Research grant Cunemark, Researcher, Research grant; Brainsway, Researcher, Research grant.

Naomi Fineberg has the following disclosures: European College of Neuropsychopharmacology, Researcher, Research support; British Association for Psychopharmacology, Attendance at psychiatric meetings, Financial support; National Institute of Health Research, Researcher, Research support Informa, Associate Editor, Fees; Taylor and Francis, Associate Editor, Royalties. 
Dan Stein has the following disclosures: Ambrf, Consultant, Research grants and/or consultancy honoraria; Biocodex, Consultant, Research grants and/or consultancy honoraria; Cipla, Consultant, Research grants and/or consultancy honoraria; Lundbeck, Consultant, Research grants and/or consultancy honoraria Novartis, Consultant, Research grants and/or consultancy honoraria, Servier, Consultant, Research grants and/or consultancy honoraria; Sun, Consultant, Research grants and/or consultancy honoraria; National Responsible Gambling Foundation, Consultant, Research grants and/ or consultancy honoraria.

Stefano Pallanti does not have anything to disclose.

Michael Van Ameringen has the following disclosures: Astra Zeneca, Consultant, Consulting fee; Biovail, Consultant, Consulting fee; Eli Lilly, Consultant, Consulting fee; Lundbeck, Consultant, Consulting fee; Pfizer, Consultant, Consulting fee; Servier, Consultant, Consulting fee; GlaxoSmith Kline, Speaker, Speaker fee.

Christine Lochner has the following disclosures: National Research Foundation of South Africa, Research, Research support.

Georgi Hranov does not have anything to disclose.

Oguz Karamustafalioglu does not have anything to disclose.

Luchezar Hranov has the following disclosures: Medochemie, Speaker, Honorarium; Lundbeck, Speaker and Principal investigator, Speaker's fees; and support for congresses; Actavis, Speaker, Speaker's fees; and support for congresses; National Association of General Practitioners in Bulgaria, Speaker, Speaker's fee; Eli Lilly, Speaker, Speaker's fee; Novel, Speaker, Honorarium and support for congresses; Clintara, Consultant, Honorarium; IMS, Consultant, Honorarium; EGRIS, Principal investigator, Investigator's fee; ECNP, Ambassador for BG, Support for congresses; Pfizer, Principal investigator, Investigator's fee; Merck, Principal investigator, Investigator's fee.

Joseph Zohar has the following disclosures: Lundbeck, Consultant, Consulting fees; Pfizer, Consultant, Consulting fees; Servier, Consultant, Consulting fees; Abbott, Consultant, Consulting fees; AstraZeneca, Consultant, Consulting fees; Roche, Consultant, Consulting fees.

Damiaan Denys does not have anything to disclose.

A. Carlo Altamura does not have anything to disclose. Jose M. Menchon has the following disclosures: $A B$ Biotics, Consultant, Honoraria; Medtronic, Speaker, Honoraria.

\section{REFERENCES:}

1. Rüther T, Bobes J, De Hert M, et al. EPA guidance on tobacco dependence and strategies for smoking cessation in people with mental illness. Eur Psychiatry. 2014; 29(2): 65-82.

2. Centers for Disease Control and Prevention (CDC). Vital signs: current cigarette smoking among adults aged $\geq 18$ years-United States, 2005-2010. MMWR Morb Mortal Wkly Rep. 2011; 60(35): 1207-1212.

3. Bronisch T, Höfler M, Lieb R. Smoking predicts suicidality: findings from a prospective community study. J Affect Disord. 2008; 108 (1-2): 135-145.

4. Bejerot $\mathrm{S}$, Humble M. Low prevalence of smoking in patients with obsessive-compulsive disorders. Compr Psychiatry. 1999; 40(4): 268-272.

5. Bejerot S, von Knorring L, Ekselius L. Personality traits and smoking in patients with obsessive-compulsive disorder. Eur Psychiatry. 2000; 15(7): 395-401.

6. Abramovitch A, Pizzagalli DA, Geller DA, Reuman L, Wilhelm S. Cigarette smoking in obsessive-compulsive disorder and unaffected parents of OCD patients. Eur Psychiatry. 2015; 30(1): 137-144.

7. Dell'Osso B, Benatti B, Buoli M, et al. The influence of age at onset and duration of illness on long-term outcome in patients with obsessive-compulsive disorder: a report from the International College of Obsessive Compulsive Spectrum Disorders (ICOCS). Eur Neuropsychopharmacol. 2013; 23(8): 865-871.

8. Yen JY, Ko CH. The association between internet gaming disorder and nicotine dependence: the mediating role of impulsivity. Alcohol Alcohol. 2014; 49: i69.

9. Hollander E, Kim S, Braun A, Simeon D, Zohar J. Cross-cutting issues and future directions for the OCD spectrum. Psychiatry Res. 2009; 170(1): 3-6.

10. Angelakis I, Gooding P, Tarrier N, Panagioti M. Suicidality in obsessive compulsive disorder (OCD): a systematic review and meta-analysis. Clin Psychol Rev. 2015; 39: 1-15. 